\title{
Strong Near-infrared Spectral Variability of the Young Cloudy L Dwarf Companion VHS J1256-1257 b
}

\author{
Brendan P. Bowler ${ }^{1}$ (D), Yifan Zhou ${ }^{2,7}$, Caroline V. Morley ${ }^{1}$ (D), Tiffany Kataria ${ }^{3}$ (iD, Marta L. Bryan ${ }^{4}$ (i), Björn Benneke ${ }^{5}$ (D), and \\ Konstantin Batygin ${ }^{6}$ (D) \\ ${ }^{1}$ Department of Astronomy, The University of Texas at Austin, Austin, TX 78712, USA; bpbowler@astro.as.utexas.edu \\ 2 McDonald Observatory and the Department of Astronomy, The University of Texas at Austin, Austin, TX 78712, USA \\ ${ }^{3}$ Jet Propulsion Laboratory, California Institute of Technology, 4800 Oak Grove Drive, Pasadena, CA, USA \\ ${ }^{4}$ Department of Astronomy, 501 Campbell Hall, University of California Berkeley, Berkeley, CA 94720-3411, USA \\ ${ }^{5}$ University of Montreal, Montreal, QC H3T 1J4, Canada \\ ${ }^{6}$ Division of Geological and Planetary Sciences, California Institute of Technology, Pasadena, CA 91125, USA \\ Received 2020 January 27; revised 2020 March 18; accepted 2020 March 19; published 2020 April 17
}

\begin{abstract}
Rotationally modulated variability of brown dwarfs and giant planets provides unique information about their surface brightness inhomogeneities, atmospheric circulation, cloud evolution, vertical atmospheric structure, and rotational angular momentum. We report results from Hubble Space Telescope/Wide Field Camera 3 near-infrared time-series spectroscopic observations of three companions with masses in or near the planetary regime: VHS J125601.92-125723.9 b, GSC 6214-210 B, and ROXs 42 B b. VHS J1256-1257 b exhibits strong total intensity and spectral variability with a brightness difference of $19.3 \%$ between 1.1 and $1.7 \mu \mathrm{m}$ over $8.5 \mathrm{hr}$ and even higher variability at the $24.7 \%$ level at $1.27 \mu \mathrm{m}$. The light curve of VHS J1256-1257 b continues to rise at the end of the observing sequence so these values represent lower limits on the full variability amplitude at this epoch. This observed variability rivals (and may surpass) the most variable brown dwarf currently known, 2MASS J21392676 +0220226 . The implied rotation period of VHS J1256-1257 b is $\approx 21-24 \mathrm{hr}$ assuming sinusoidal modulations, which is unusually long for substellar objects. No significant variability is evident in the light curves of GSC $6214-210 \mathrm{~B}(<1.2 \%)$ and ROXs $42 \mathrm{~B}$ b $(<15.6 \%)$. With a spectral type of L7, an especially red spectrum, and a young age, VHS J1256-1257 b reinforces emerging patterns between high variability amplitude, low surface gravity, and evolutionary phase near the $\mathrm{L} / \mathrm{T}$ transition.
\end{abstract}

Unified Astronomy Thesaurus concepts: Brown dwarfs (185); Exoplanet atmospheric variability (2020)

\section{Introduction}

Time series photometry and spectroscopy of brown dwarfs has opened up a new window into the physical properties and atmospheric structure of substellar objects. Over the past decade, high-precision infrared monitoring programs have demonstrated that most brown dwarfs are variable at the $0.2 \%-5 \%$ level between 1 and $5 \mu \mathrm{m}$, especially those spanning the $\mathrm{L}$ and $\mathrm{T}$ spectral classes between $\approx 500$ and $2500 \mathrm{~K}$ (e.g., Artigau et al. 2009; Apai et al. 2013; Radigan et al. 2014; Metchev et al. 2015). There is now abundant evidence that the primary source of this variability is from rotationally modulated surface features in the form of evolving heterogeneous condensate clouds (e.g., Showman \& Kaspi 2013; Crossfield et al. 2014), at least among low-temperature late-L and T-type brown dwarfs, analogous to Jupiter's latitudinally banded structure where gaps in the cloud deck result in bright regions at infrared wavelengths (e.g., Antuñano et al. 2019; Ge et al. 2019). These patchy clouds produce hot and cold spots which, coupled with rotation, result in periodic disk-integrated brightness variations that evolve in amplitude, phase, and wavelength (Buenzli et al. 2012; Apai et al. 2017).

Most of these monitoring campaigns have focused on old (several Gyr) brown dwarfs in the field. Recently there has been increased interest in exploring the variability properties of both young brown dwarfs and giant planets found with highcontrast imaging to examine the influence of surface gravity on variability properties. For example, Biller et al. (2015) found

\footnotetext{
McDonald Prize Fellow.
}

that the $\approx 8 M_{\text {Jup }}$ object PSO J318.5-22 exhibits strong variability at the $10 \%$ level in $J$ band. Lew et al. (2016) reported that the young isolated brown dwarf WISE J0047 +6803 is highly variable from 1.1 to $1.7 \mu \mathrm{m}$, with peak-topeak brightness changes as high as $8 \%$. Variability has also been confidently observed in a growing number of companions at or below the deuterium-burning limit $\left(\approx 13 M_{\text {Jup }}\right)$ : $2 \mathrm{M} 1207-$ 3932 b (Zhou et al. 2016), Ross 458 C (Manjavacas et al. 2019), HD 203030 B (Miles-Paez et al. 2019), HN Peg B (Zhou et al. 2018), 2M0122-2439 b (Zhou et al. 2019), and GU Psc b (Naud et al. 2017; Lew et al. 2020).

Several statistical patterns are emerging from variability surveys of substellar objects. There is evidence that low-gravity brown dwarfs have higher variability amplitudes compared to their higher-gravity counterparts (Metchev et al. 2015). Vos et al. (2018) found that young L dwarfs have higher intrinsic rates of variability compared to field brown dwarfs with $98 \%$ confidence, an indication that low surface gravity plays an important role in shaping the spatial distribution and physical properties of condensate clouds. Viewing geometry also appears to impact these observational signatures; brightness changes are most strongly attenuated for brown dwarfs observed at low inclinations (closer to pole-on orientations; Vos et al. 2017).

Extending variability studies to directly imaged exoplanets is challenging because of their high contrasts and close separations. Over the past decade, a steadily growing population of giant planets located at unexpectedly wide separations of tens to hundreds of astronomical units from their host stars has been 
identified. Their relatively wide separations $(>100 \mathrm{au})$ and modest contrasts $(<10 \mathrm{mag})$ make them amenable to detailed photometric and spectroscopic observations. Here we present results from a Hubble Space Telescope (HST) program to obtain time-series spectroscopic light curves of the three lowmass companions VHS J125601.92-125723.9 b (hereafter VHS J1256-1257 b), GSC 6214-210 B, and ROXs $42 \mathrm{~B}$ b. Our Wide-Field Camera 3 (WFC3) G141 grism observations span 1.1-1.7 $\mu \mathrm{m}$, enabling simultaneous monitoring at $J$-band, $H$-band, and over the $1.4 \mu \mathrm{m}$ water feature. None of these companions have previously been monitored for variability.

VHS J1256-1257 b is an unusually red L7 companion orbiting a young binary brown dwarf at 8!' 1 (Gauza et al. 2015; Stone et al. 2016). Depending on the system's age and distance, which remain poorly constrained, the companion's luminosity implies a lower mass limit between 11 and $26 M_{\text {Jup }}$ (Rich et al. 2016). The $L$-band spectrum of VHS J1256-1257 b from Miles et al. (2018) shows signs of weak methane absorption and thick photospheric clouds. GSC 6214-210 B is an M9.5 companion with a mass of $\approx 14 M_{\text {Jup }}$ orbiting at 2 !" $(240 \mathrm{au})$ from its young ( $\approx 17 \mathrm{Myr}$ ) Sun-like host star (Ireland et al. 2011; Bowler et al. 2014; Pearce et al. 2019). ROXs 42 B b has a mass of $\approx 10 M_{\mathrm{Jup}}$ and a near-infrared spectral type of L1 (Bowler et al. 2014; Currie et al. 2014; Kraus et al. 2014). It is the most challenging target in our sample to observe with HST because of its close angular separation to its host star (1"!2, or $140 \mathrm{au})$. All three companions have rotational broadening measurements from high-resolution near-infrared spectroscopy (Bryan et al. 2018).

This Letter is organized as follows. In Section 2 we describe the HST observations, spectral extraction, and corrections for detector ramp effects. In Section 3 we discuss the companion light curves and interpretation of variability observed in VHS J1256-1257 b. Our observations and results are summarized in Section 4.

\section{Observations}

Time-series spectroscopic monitoring was carried out with HST's WFC3/IR camera with the G141 near-infrared grism $(\lambda \lambda=1.1-1.7 \mu \mathrm{m} ; \lambda / \delta \lambda \approx 130)$ on UT 2018 March 5-6, UT 2018 March 14-15, and UT 2018 June 14 for VHS J1256-1257 b, ROXs 42 B b, and GSC 6214-210 B, respectively (HST Proposal GO-15197). The spectral grasp of our observations samples a range of atmospheric pressure levels and prominent molecular absorption features, most notably the water feature centered at $1.4 \mu \mathrm{m}$ and methane beyond $1.6 \mu \mathrm{m}$. Our program was designed to span $8.5 \mathrm{hr}$ over six contiguous orbits for each target with unobservable gaps of about 45 minutes during each orbit. Observations were carried out in nominal stare mode with the telescope roll angle constraints oriented so that the grism dispersion direction was approximately orthogonal to the star-planet position angle.

At the start of each orbit, several direct images were taken in the $F 132 N$ filter to determine the location of the companion and to calibrate the wavelength solution. The $256 \times 256$ subarray $\left(\approx 30^{\prime \prime} \times 30^{\prime \prime}\right.$ field of view) was read out to reduce buffer dumps and improve readout time efficiency. Exposure times and number of non-destructive reads were chosen to avoid saturating the host star at the location of the companion and to ensure adequate signal-to-noise ratio of the companion spectrum. No dithering was carried out to mitigate flat-fielding errors.
We acquired 11 grism images of VHS J1256-1257 b per orbit for a total of 66 frames, each with an exposure time of $224 \mathrm{~s}$ and a SPARS25, NSAMP $=11$ readout sequence. For ROXs 42 B b, 21 grism images were taken for the first orbit followed by 22 for orbits $2-6$, totaling 131 images altogether. The integration time was $103 \mathrm{~s}$ per image and the SPARS10, NSAMP $=15$ sequence was chosen. 137 grism images were obtained for GSC 6214-210 B: 22 were acquired in orbit 1 followed by 23 frames during orbits 2-6. Each had an integration time of $103 \mathrm{~s}$ with SPARS10, NSAMP $=15$.

\subsection{Data Reduction}

We adopt different strategies to extract the grism spectra of our three targets. For VHS J1256-1257 b, the contamination from the host star spectral trace at the location of the companion (a separation of 57.7 pixels) is negligible: the average flux level at the location of VHS J1256-1257 b is about $0.23 \mathrm{e}^{-} \mathrm{s}^{-1}$ pixel $^{-1}$, which is less than $20 \%$ of the average sky background. We therefore do not carry out pointspread function (PSF) subtraction for this system. For the other two targets, the contamination from their host stars is much more significant and requires PSF subtraction in the dispersion direction to recover the companions. Below we describe details of the data reduction and spectral extraction procedure for each system in this program.

\subsubsection{VHS J1256-1257 b}

Basic image reduction is carried out with STScI's calwfc3 pipeline, which performs bias correction, linearity correction, dark subtraction, unit/gain conversion, and cosmic ray identification with up-the-ramp fitting. We extract individual spectra from the $f t$ images with our custom WFC3/IR timeresolved spectroscopic pipeline, which makes use of the aXe slitless spectral extraction package (Kümmel et al. 2009) and has been employed in multiple studies to measure rotational modulations of brown dwarfs (e.g., Buenzli et al. 2012, 2014; Apai et al. 2013; Zhou et al. 2018). As part of this pipeline, we first expand the $256 \times 256$ subarray to the $1014 \times 1014$ fullarray images so that they are compatible with aXe. We then identify bad pixels with data quality flag 4 (indicating a bad detector pixel), 16 (hot pixel), 32 (unstable response), and 256 (full-well saturation) and correct them with bi-linear interpolation. We also search and correct for pixels that are affected by cosmic rays but not corrected by the up-the-ramp fit. These pixels are identified by comparing the time sequence of each individual pixel to its median-filtered light curve (in a window size of 5 pixels) and select $7 \sigma$ outliers. These pixels are then corrected through bi-linear interpolation with adjacent unaffected pixels. We develop and implement our own sky subtraction routine following Brammer et al. (2015) to incorporate and optimize recently updated multi-component WFC3 master sky images, and thus run the axeprep task with the "background" option turned off. Then the axecore task is executed with a 4 pixel radius window to extract spectra.

For the VHS J1256-1257 b sequence, three spectra of VHS J1256-1257 b, the host VHS J1256-1257 AB (which is unresolved here), and the background star 2MASS J12560179-1257390 are extracted from each grism image (Figure 1). This results three spectroscopic light curves in units of both count rate $\left(\mathrm{e}^{-} \mathrm{s}^{-1}\right)$ and flux density (erg $\mathrm{s}^{-1}$ $\mathrm{cm}^{-2} \mu \mathrm{m}^{-1}$ ), as well as their corresponding measurement 

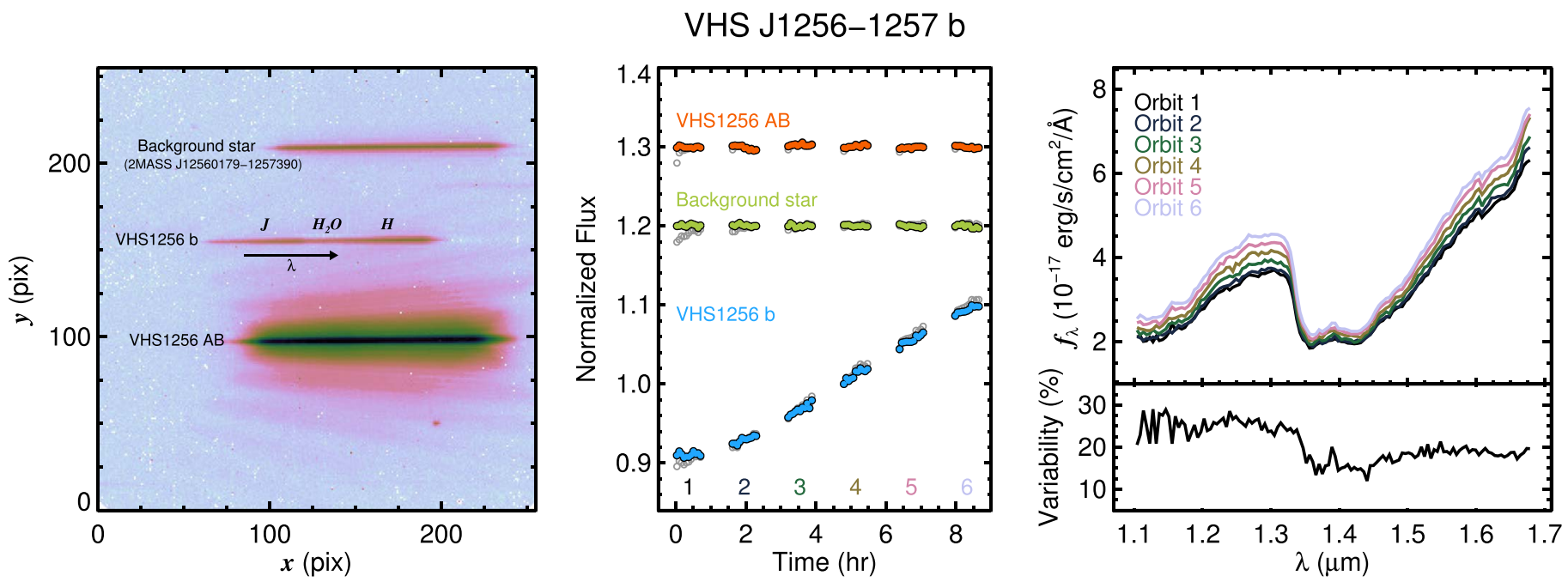

Figure 1. HST time-series grism observations of VHS J1256-1257 b. Left panel: grism image of VHS J1256-1257 AB, VHS J1256-1257 b, and the nearby background star 2MASS J12560179-1257390. Wavelength increases to the right along the dispersion direction. Middle panel: extracted and normalized light curves of all three objects from 1.1 to $1.68 \mu \mathrm{m}$. VHS J1256-1257 AB and the nearby background star are approximately constant over all six orbits $(\approx 8.5 \mathrm{hr})$, whereas $\mathrm{VHS}$ J1256-1257 b clearly shows a substantial increase in brightness. Gray open circles represent the uncorrected photometry; colored symbols show the normalized flux after correcting for ramp effects using our RECTE pipeline (see Section 2.1 for details). Orbits are labeled at the bottom of the panel. Right panel: median spectra for each orbit (top section) and relative spectral variability ( $\max / \mathrm{min}-1$; bottom section). Variability is strongest in $J$-band and weakest in the $1.4 \mu \mathrm{m}$ water band.
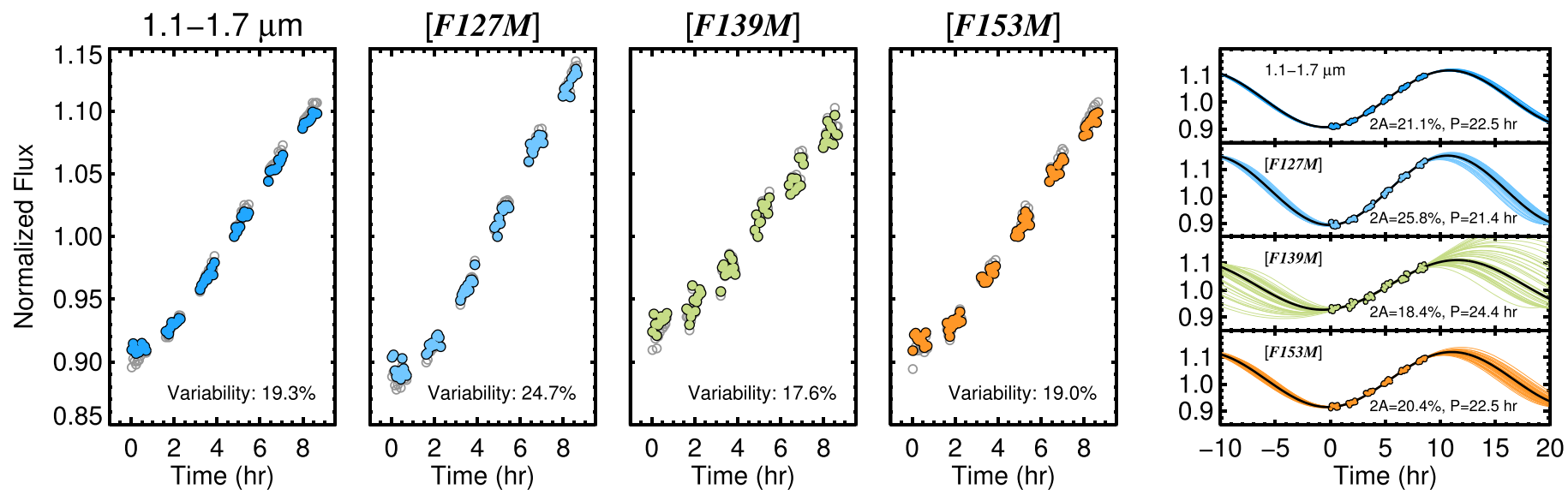

Figure 2. HST near-infrared spectroscopic light curves of VHS J1256-1257 b. Time-series photometry represent the full 1.1-1.68 $\mu \mathrm{m}$ region as well as synthesized $F 127 M, F 139 M$, and $F 153 M$ bandpasses from left to right. Variability amplitudes are strongly wavelength dependent. The highest amplitude (24.7\%) is seen in $F 127 M$ band with $F 139 M$ and $F 153 M$ bands being lower (17.6\% and $19.0 \%$, respectively). The spectroscopic light curve continues to rise at the end of the observations so these values are likely lower limits for this epoch. Sinusoidal fits to each light curve are shown in the rightmost panel. Displayed are 50 random draws from the parameter posteriors.

uncertainties. We then remove the "ramp-effect" systematics in the light curves (e.g., Berta et al. 2012). Here we use the Ramp Effect Charge Trapping Eliminator (RECTE; Zhou et al. 2017) tool to model and correct these systematics. RECTE estimates the "ramp-effect" profile by assuming the systematics are introduced by charge trapping and delayed release due to detector defects. We adopt a set of pre-determined parameters that describe the charge trap numbers, efficiency, and trapping timescales. The remaining parameter that primarily determines the systematic profile is the pixel illumination level. We input the median-combined images to the RECTE model and calculate the ramp profile for each pixel, then sum the profiles of the same image columns to identify the correction term for each wavelength. Our final spectroscopic light curves are obtained by dividing the correction term from the raw extracted spectra.

Light curves of VHS J1256-1257 b, the host binary, and the background star are measured by integrating the spectra in the wavelength dimension. Four light curves are produced for each object: a broadband curve spanning $1.10-1.68 \mu \mathrm{m}$ where the G141 grism throughput is above $30 \%$, as well as time series photometry synthesized in the $F 127 M$ bandpass, $F 139 M$ bandpass, and $F 153 M$ bandpass (Figure 2). The synthesized light curves are measured by convolving each spectrum with HST transmission curves and then integrating the result. Note that the red cutoff of the $G 141$ grism truncates the traditional MKO $H$ bandpass by about $0.1 \mu \mathrm{m}$, so we chose to use medium-band HST filters instead of standard near-infrared filters.

\subsubsection{GSC 6214-210 B and ROXs $42 \mathrm{~B} \mathrm{~b}$}

GSC 6214-210 B and ROXs $42 \mathrm{~B}$ b are both embedded in the PSF wings of their host stars in the grism images. For these observations, our strategy is to subtract the spectral PSF of the primary star in the dispersion direction before spectral 

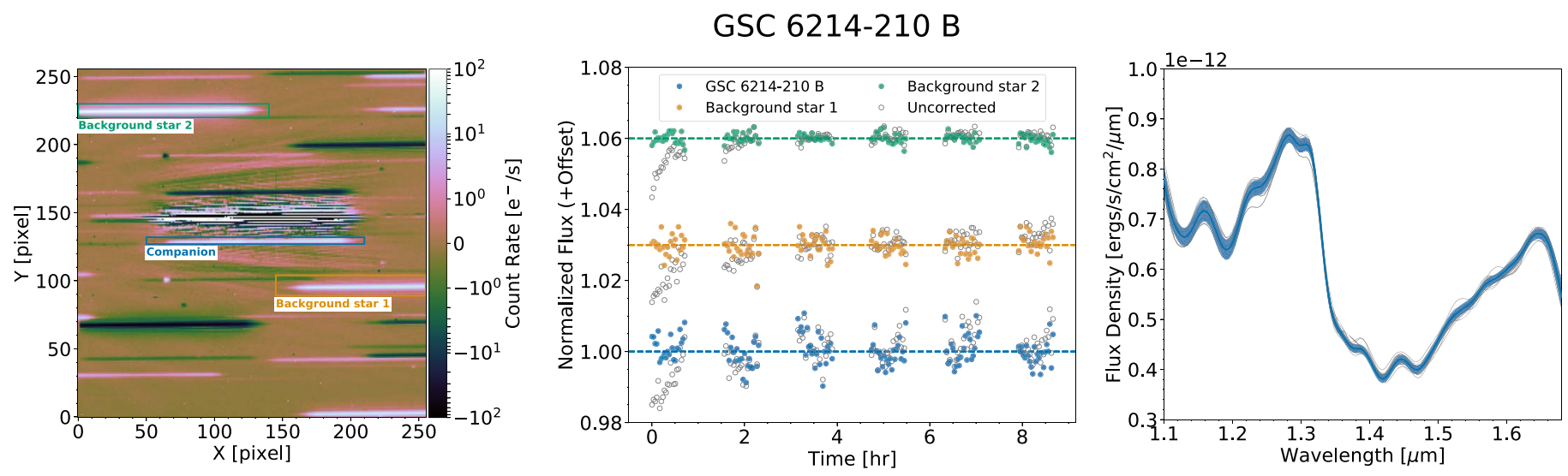

ROXs 42 B b
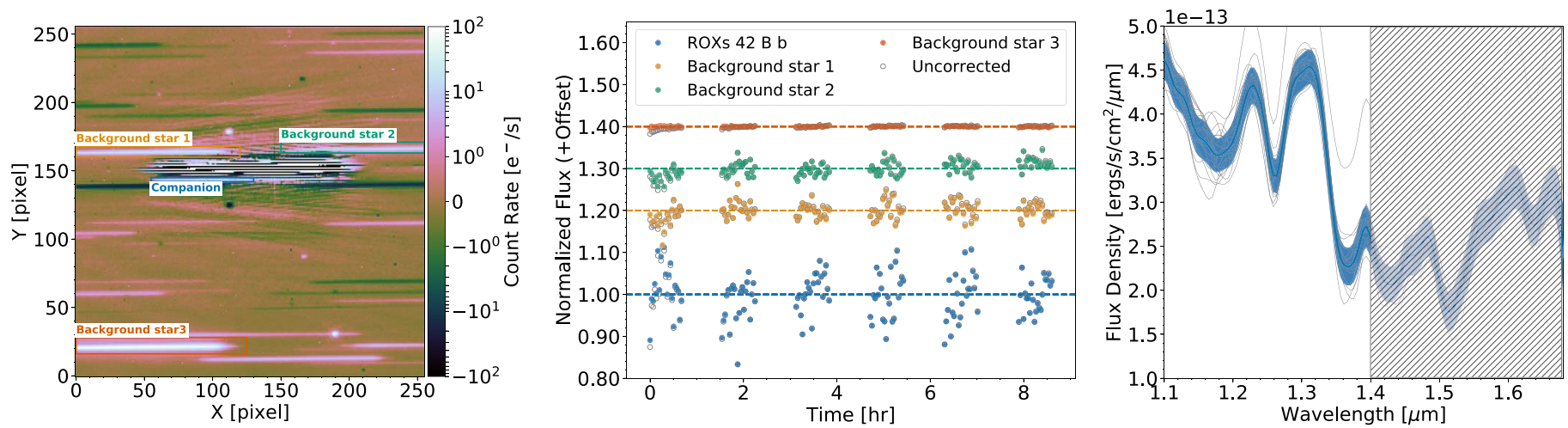

Figure 3. Results from HST time-series grism observations of GSC 6214-210 B (top row) and ROXs 42 B b (bottom row). Left panels: grism images of each system. PSF subtraction of the host star is carried out using the mirrored image about the central axis, which results in "dipole spectra" of the companion and background stars. The dispersion direction is nearly aligned with the $x$-axis, with wavelength increasing to the right. Middle panels: extracted light curves of the companions and nearby background stars. Gray open circles represent the uncorrected photometry; colored symbols show the normalized flux (plus arbitrary offsets) after correcting for detector ramp effects. No modulations are evident for either GSC 6214-210 B $(<1.2 \%$; $3 \sigma$ upper limit) or ROXs $42 \mathrm{~B}$ b ( $<15.6 \%$; $3 \sigma$ upper limit). Right panels: extracted spectra of each system. For ROXs $42 \mathrm{~B}$ b, wavelengths beyond about $1.4 \mu \mathrm{m}$ are heavily contaminated by the host star and are not reliable.

extraction. By assuming that the spectral trace is symmetric with respect to the central axis, we mirror the image about this axis and subtract it individually from each science frame (Figure 3 ). Because the spectral trace is tilted by $\sim 0.6$ with respect to the image $x$-axis, the mirrored image is first rotated to match the original primary spectral PSF. To optimize the subtraction, we set the template scaling parameter and the tilt angle as free parameters and fit them to minimize the rms subtraction residuals surrounding the companion spectral traces. Following PSF subtraction, the steps to extract the spectra and correct the light curves for these companions and several nearby background stars in the images are identical to the procedure for VHS J1256-1257 b.

\section{Results and Discussion}

No variability is observed in the observations of GSC 6214$210 \mathrm{~B}$ and ROXs $42 \mathrm{~B} \mathrm{~b}$, but significant evolution is readily apparent for VHS J1256-1257 b. The brightness difference for VHS J1256-1257 b is unusually large compared to the 28 strong variables with measured amplitudes greater than $2 \%$ (Figure 4). Metchev et al. (2015) found that most brown dwarfs exhibit low-amplitude variability $(\approx 0.2 \%-2 \%)$, but strong variability at the $2 \%-10 \%$ level is rarer, especially outside the L/T transition (Radigan 2014; Eriksson et al. 2019). Only five brown dwarfs are known to exhibit exceptionally highamplitude variability above the $10 \%$ level (see Eriksson et al.
2019 for a recent compilation): PSO J318.5-22 (L7; Biller et al. 2018; Vos et al. 2018), WISE J104915.57-531906.1 B (T0.5; Biller et al. 2013; Gillon et al. 2013; Buenzli et al. 2015) 2MASS J22153705+2110554 (T1; Eriksson et al. 2019), 2MASS J13243553+6358281 (T2; Yang et al. 2016), and 2MASS J21392676+0220226 (T1.5; e.g., Radigan et al. 2012). The brightness difference of $24.7 \%$ we measure in the synthesized F127M filter for VHS J1256-1257 b is the secondhighest after 2MASS J21392676+0220226, which exhibits exceptionally strong variability up to $27 \%$ in the $J$-band (Apai et al. 2013). Among these six strong variables (amplitudes $>10 \%$ ), all have spectral types between L7 and T2 and three show spectroscopic and/or kinematic evidence of youth (VHS J1256-1257 b, PSO J318.5-22, and 2MASS J13243553 +6358281). These same three objects have masses near or within the planetary regime, supporting trends of highamplitude modulation and low surface gravity found by Metchev et al. (2015) and Vos et al. (2018).

The large brightness changes from VHS J1256-1257 b exhibit inflection points midway through the observations (Figure 2), which resemble sinusoidal modulations commonly observed among brown dwarfs and planetary-mass objects. To estimate the rotation period and total variability amplitude, we fit the normalized broad-band light curve and synthesized light curves for individual bands assuming a sinusoidal model as 


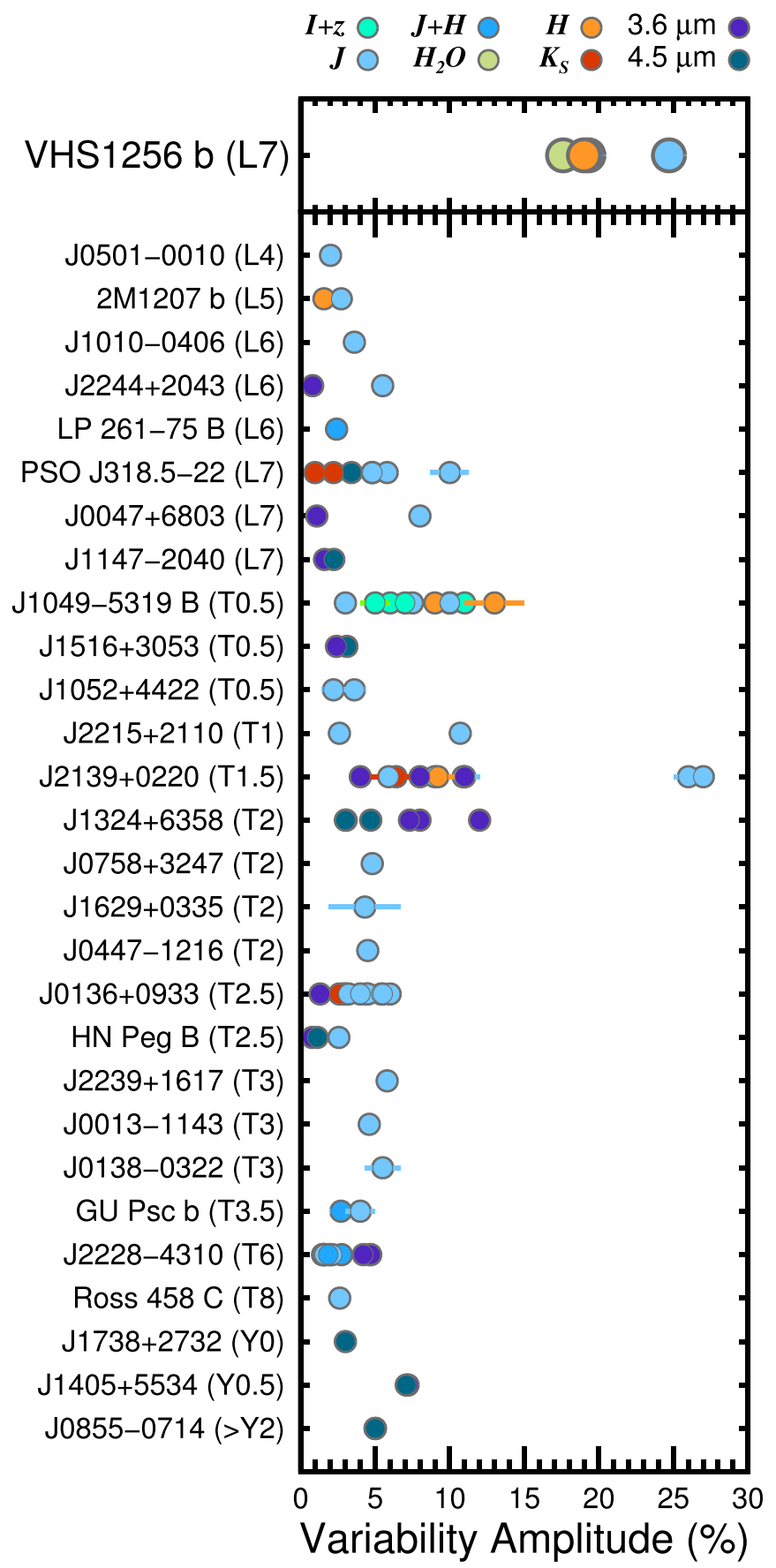

Figure 4. Amplitudes of the 28 strongest variable brown dwarfs and planetarymass objects with peak-to-peak brightness changes $>2 \%$ compared to our observations of VHS J1256-1257 b (top panel). Objects are sorted by spectral type and variability amplitudes are color coded by filter. VHS J1256-1257 b has the second largest amplitude after the T1.5 brown dwarf 2MASS J21392676+0220226 (Radigan et al. 2012; Apai et al. 2013). Data are from Eriksson et al. (2019), Manjavacas et al. (2017, for LP-261-75 B), Naud et al. (2017, for GU Psc b), and Lew et al. (2020, for GU Psc b).

follows: $f(t)=a+A \sin ((2 \pi / P) t+\phi){ }^{8}$ Here $f$ is the normalized flux, $a$ is a constant offset, $A$ is the semi-amplitude, $P$ is the period, and $\phi$ is a phase offset. Parameter posterior

\footnotetext{
8 We tested both linear and sinusoidal relations to model the curves and calculated Bayesian Information Criterion (BIC) values, where $\mathrm{BIC}=\chi^{2}+$ $k \ln N$. Here $k$ is the number of free parameters in the fit and $N$ is the number of data points (66). The $\triangle \mathrm{BIC}$ between the two models is 1133 for the broadband light curve, indicating a very strong preference for the sine model.
}

distributions are sampled using Markov Chain Monte Carlo with a Metropolis-Hastings algorithm. Step sizes for trial values are chosen so that acceptance rates are between $20 \%$ and $30 \%$. A uniform prior was adopted in $a$ (from 0 to 2 ), $A$ (from 0 to 2 ), $P$ (from 0 to $100 \mathrm{hr}$ ), and $\phi$ (from 0 to $2 \pi$ ).

Results of the sinusoidal fits are shown in Figure 2. For the full $1.1-1.7 \mu \mathrm{m}$ light curve, we infer a rotation period of $22.5_{-0.4}^{+0.3} \mathrm{hr}$ and a peak-to-peak amplitude of $21.1_{-0.2}^{+0.2} \%$. The $\chi^{2}$ value of the best-fit curve is 126.1 , the BIC value is 142.9 , and the reduced $\chi^{2}$ value $\left(\chi_{\nu}^{2}=\chi^{2} /(N-k)\right)$ is 2.0. Here $k=4$ and $N=66$. The synthetic photometry for individual bands implies similar periods but significant wavelength-dependent amplitude differences. We find a period of $21.4_{-0.9}^{+0.8} \mathrm{hr}$ and a peak-to-peak amplitude of $25.8_{-0.7}^{+0.8} \%$ for $F 127 M\left(\chi^{2}=65.1\right.$, BIC $=81.9$, $\left.\chi_{\nu}^{2}=1.05\right), 24_{-5}^{+4} \mathrm{hr}$ and an amplitude of $18.4_{-2.8}^{+2.2} \%$ for $F 139 \mathrm{M}$ $\left(\chi^{2}=67.2, \mathrm{BIC}=84.0, \chi_{\nu}^{2}=1.08\right)$, and $22.5_{-0.9}^{+1.0} \mathrm{hr}$ and an amplitude of $20.4_{-0.7}^{+0.6} \%$ for $F 153 M\left(\chi^{2}=81.4\right.$, BIC $=98.2$, $\left.\chi_{\nu}^{2}=1.23\right)$. The implied rotation period of $\approx 21-24 \mathrm{hr}$ assumes the long-term variability is smooth and periodic. It also neglects any possible latitudinal dependence of the rotation rate, for example if the signal originates from non-equatorial features where banding or differential rotation could produce a longer variability period than at equatorial regions. These results highlight how unusual the properties of VHS J1256-1257 b are in terms of its variability amplitude, its strong chromatic modulations, and its long rotation period compared to typical values of $\approx 3-20 \mathrm{hr}$ for brown dwarfs (e.g., Biller 2017). We note that this long rotation period can only be reconciled with the projected rotational velocity of $13.5_{-4.1}^{+3.6} \mathrm{~km} \mathrm{~s}^{-1}$ from Bryan et al. (2018) with a large physical radius and (nearly) edge-on viewing geometry. The inferred inclination distribution of VHS J1256-1257 b is explored in more detail in a companion study (Y. Zhou et al. 2020, in preparation).

Spectroscopic observations also enable searches for wavelength-dependent phase lags, which provide information about the nature and distribution of surface features causing the brightness modulations. These light curve phase shifts have been observed in several objects, especially when comparing simultaneous near-infrared and mid-infrared light curves (e.g., Buenzli et al. 2012; Biller et al. 2018), but this phenomenon is not universal among variable brown dwarfs. We find phases of $4.73_{-0.04}^{+0.04} \mathrm{rad}, 4.89_{-0.16}^{+0.15} \mathrm{rad}$, and $4.77_{-0.05}^{+0.05} \mathrm{rad}$ from our sinusoidal fits to the $F 127 M, F 139 M$, and $F 153 M$ light curves of VHS J1256-1257 b, respectively. The phase for the full $1.1-1.7 \mu \mathrm{m}$ region is $4.84_{-0.02}^{+0.02} \mathrm{rad}$. We conclude that there is no significant evidence for distinct phase lags from these observations.

The observed variability of brown dwarfs has largely been attributed to the presence of clouds, which can produce a variety of time-dependent observable signatures. This is expected to be especially pronounced near the $\mathrm{L} / \mathrm{T}$ transition as brown dwarfs cool and cloud decks sink below the photosphere at lower effective temperatures. At higher altitudes (low pressures), patchy clouds tend to produce gray modulations with little change in variability amplitude as a function of wavelength. When located at lower altitudes (higher pressures), clouds can introduce a wavelength-dependence to the variability amplitude (e.g., Buenzli et al. 2012). In this scenario, spectral regions that probe deeper into the atmosphere (such as the $J$-band) will be more susceptible to variability compared to wavelengths sensitive to higher altitudes (such as the $1.4 \mu \mathrm{m}$ water band). These wavelength-dependent amplitude 


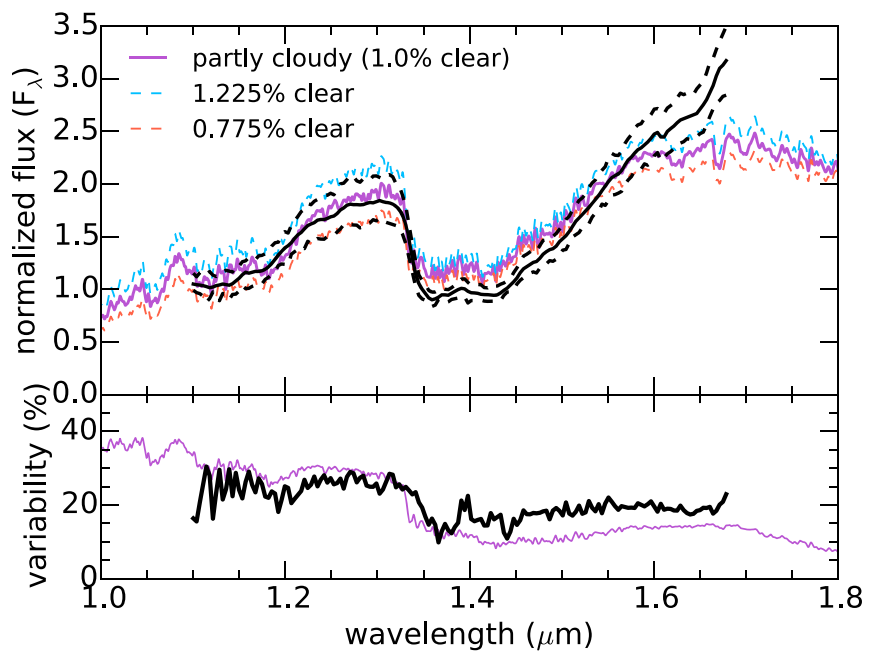

Figure 5. Simulated spectra used to represent atmospheric variability. By assuming that the planet has partial cloud coverage, we are able to reproduce the emergent spectrum (with model parameters $T_{\text {eff }}=900 \mathrm{~K}, \log g=4.0$, and $f_{\text {sed }}=0.3$; top section), the variability amplitude, and the wavelengthdependent variability behavior (bottom section) using a $1 \%$ clear surface with $0.225 \%$ changes to the cloud-covering fraction. Dashed lines in the top section show the minimum and maximum variability of the data (black) and model (orange and light blue).

differences are therefore generally interpreted as signatures of mid-altitude clouds and have predominantly been observed in early $\mathrm{T}$ dwarfs, whereas gray (wavelength-independent) variability likely arises from high-altitude clouds and appears to be more common among mid-L dwarfs (e.g., Yang et al. 2015; Artigau 2018). VHS J1256-1257 b has a spectral type of L7 but exhibits unusually strong relative spectral variability of $5.7 \%$ between $F 127 M$ and $F 153 M$, and even larger differences of $7.1 \%$ between $F 127 M$ and $F 139 M$. Aside from a small increase at $\approx 1.39 \mu \mathrm{m}$ in the middle of the water band, these changes are relatively constant within each bandpass (Figure 1). In this respect VHS J1256-1257 b more closely resembles the spectral variability behavior of early $\mathrm{T}$ dwarfs than mid-L dwarfs.

VHS J1256-1257 b also shares similarities with the two other low-mass variable objects WISE J0047+6803 and PSO J318.5-22: all have comparable spectral types (L6-L7), extremely red near-infrared slopes $\left(J-K_{S}=2.5-2.8 \mathrm{mag}\right)$, and similar wavelength-dependent variability patterns from 1.1-1.7 $\mu \mathrm{m}$ with the strongest variability at the $J$-band and the weakest variability in the $1.4 \mu \mathrm{m}$ water band (Lew et al. 2016; Biller et al. 2018). However, these traits are all significantly enhanced in VHS J1256-1257 b, which may be a result of thinner high-altitude clouds or distinct dust properties compared to the other two objects.

To provide physical insight into the observed variability, partly cloudy model spectra were generated following the methodology presented in Marley et al. (2010) and Morley et al. (2014). All models use $T_{\text {eff }}=900 \mathrm{~K}$ and $\log g=4.0$ and assume that the atmosphere is in chemical and radiativeconvective equilibrium. A small grid of sedimentation efficiency $\left(f_{\text {sed }}\right)$ and cloud-free surface fraction $\left(f_{\mathrm{h}}\right)$ was considered $\left(f_{\text {sed }}=0.3-2 ; f_{\mathrm{h}}=1 \%-10 \%\right)$; very lofted clouds $\left(f_{\text {sed }}=0.3\right)$ with a small cloud-free surface fraction $(1 \%)$ provided the best match, as expected for this red, dusty object. After converging this partly cloudy model, moderate resolution spectra through the cloudy and clear columns were generated
(Figure 5). The trend and amplitude in variability can be matched by varying the cloud-covering fraction by $0.225 \%$ (from $0.775 \%$ cloud-free to $1.225 \%$ cloud-free).

The resulting model is meant to demonstrate that the overall trend-higher amplitude variability in the $J$-band relative to the water band and the $H$-band-and large amplitude can be matched by considering non-uniform clouds. Future work should investigate disequilibrium chemistry (a likely cause of the model-data discrepancy in the $H$-band); provide detailed fits to larger grids of model spectra; and investigate different cloud treatments (e.g., thicker and thinner clouds and latitudinal banding rather than thick clouds and cloud-free regions)particularly using 2D or 3D models.

\subsection{Upper Limits on Variability for GSC 6214-210 B and ROXs $42 B b$}

In contrast, our light curves of GSC 6214-210 B and ROXs $42 \mathrm{~B} \mathrm{~b}$ appear to be flat with no evidence of significant variability. The photometric precision for these companions is limited by the PSF subtraction in the grism images. This procedure was substantially more challenging for ROXs $42 \mathrm{~B} \mathrm{~b}$ owing to the close separation of its host star (1".2). We measure rms levels of $0.4 \%$ for GSC $6214-210 \mathrm{~B}$, or a $3 \sigma$ upper limit of $1.2 \%$. For ROXs $42 \mathrm{~B}$ b we find an rms of $5.2 \%$, or a $3 \sigma$ upper limit of $15.6 \%$.

\section{Summary}

We have obtained HST/WFC3 near-infrared time-series spectroscopy of the three young companions VHS J1256-1257 b, GSC 6214-210 B, and ROXs 42 B b, all of which have masses at or near the deuterium-burning boundary. Results from this program are summarized below.

1. Our $8.5 \mathrm{hr}$ observations show strong brightness changes in VHS J1256-1257 b at the level of $19.3 \%$ across the full $1.1-1.68 \mu \mathrm{m}$ region. For the synthesized bandpasses we find $24.7 \%$ variability in $F 127 M, 17.6 \%$ variability in $F 139 M$, and $19.0 \%$ variability in $F 153 M$. These are the highest variability measurements of any substellar object after the T1.5 brown dwarf 2MASS J21392676 +0220226 . The evolving light curve of VHS J1256-1257 b was still rising at the end of the observations, implying the full variability amplitude is likely to be even larger at this epoch.

2. We interpret these substantial wavelength-dependent variability amplitudes as evidence for mid-altitude cloud decks, similar to (but much stronger than) the other young red L dwarfs WISE J0047+6803 and PSO J318.5-22. The spectral shape, large-amplitude brightness modulations, and wavelength-dependent variability can be reproduced with atmospheric models comprising a $1 \%$ clear surface coupled with $0.225 \%$ changes in cloud-covering fraction.

3. Sinusoidal fits to the light curves of VHS J1256-1257 b imply rotation periods between 21 and $24 \mathrm{hr}$ for the full 1.1-1.68 $\mu \mathrm{m}$ spectral region and individual synthesized bands. We find no evidence of phase shifts from these observations, although only about $30 \%-40 \%$ of a single rotation period appears to have been sampled.

4. No variability is observed from GSC 6214-210 B or ROXs $42 \mathrm{~B} \mathrm{~b}$ at the $<1.2 \%$ and $<15.6 \%$ levels $(3 \sigma$ upper limits), respectively. These precisions are limited 
by the close separations of these companions to their host stars $(1 !$ "2-2!!2).

The large separation of VHS J1256-1257 b from its host star (8!'1) makes it an excellent target for follow-up observations spanning longer time baselines, broader wavelength coverage, and higher spectral resolution. The high near-infrared amplitude for this object also means that it especially amenable to ground-based photometric monitoring. Altogether these results for VHS J1256-1257 b suggest that other young planetarymass companions at the L/T transition, like HR 8799 bcde, HD 95086 b, HIP 65426 b, and 2MASS J22362452 $+4751425 \mathrm{~b}$, are promising companions to search for variability.

The authors thank the referee for comments that improved this manuscript, as well as Heather Knutson and Trent Dupuy for helpful discussions. This research is based on observations made with the NASA/ESA Hubble Space Telescope obtained from the Space Telescope Science Institute, which is operated by the Association of Universities for Research in Astronomy, Inc., under NASA contract NAS 5-26555. These observations are associated with program 15197. B.P.B. acknowledges support from the National Science Foundation grant AST1909209.

Facility: HST (WFC3).

\section{ORCID iDs}

Brendan P. Bowler (i) https://orcid.org/0000-0003-2649-2288 Caroline V. Morley (i) https://orcid.org/0000-0002-4404-0456 Tiffany Kataria (ib https://orcid.org/0000-0003-3759-9080 Marta L. Bryan (iD https://orcid.org/0000-0002-6076-5967 Björn Benneke (iD https://orcid.org/0000-0001-5578-1498 Konstantin Batygin (iD https://orcid.org/0000-0002-7094-7908

\section{References}

Antuñano, A., Fletcher, L. N., Orton, G. S., et al. 2019, AJ, 158, 130 Apai, D., Karalidi, T., Marley, M. S., et al. 2017, Sci, 357, 683

Apai, D., Radigan, J., Buenzli, E., et al. 2013, ApJ, 768, 121

Artigau, E. 2018, in Handbook of Exoplanets, ed. H. J. Deeg \& J. A. Belmonte (Cham: Springer), 94

Artigau, É, Bouchard, S., Doyon, R., \& Lafrenière, D. 2009, ApJ, 701, 1534 Berta, Z. K., Charbonneau, D., Désert, J.-M., et al. 2012, ApJ, 747, 35
Biller, B. 2017, AstRv, 13, 1

Biller, B. A., Crossfield, I. J. M., Mancini, L., et al. 2013, ApJL, 778, L10

Biller, B. A., Liu, M. C., Rice, K., et al. 2015, MNRAS, 450, 4446

Biller, B. A., Vos, J., Buenzli, E., et al. 2018, AJ, 155, 95

Bowler, B. P., Liu, M. C., Kraus, A. L., \& Mann, A. W. 2014, ApJ, 784, 65 Brammer, G., Ryan, R., \& Pirzkal, N. 2015, Source-dependent Master Sky Images for the WFC3/IR Grisms, Space Telescope WFC Instrument Science Rep. 2015-17

Bryan, M. L., Benneke, B., Knutson, H. A., Batygin, K., \& Bowler, B. P. 2018, NatAs, 2, 138

Buenzli, E., Apai, D., Morley, C. V., et al. 2012, ApJL, 760, L31

Buenzli, E., Apai, D., Radigan, J., Reid, I. N., \& Flateau, D. 2014, ApJ, 782, 77 Buenzli, E., Saumon, D., Marley, M. S., et al. 2015, ApJ, 798, 127

Crossfield, I. J. M., Biller, B., Schlieder, J. E., et al. 2014, Natur, 505, 654

Currie, T., Daemgen, S., Debes, J., et al. 2014, ApJL, 780, L30

Eriksson, S. C., Janson, M., \& Calissendorff, P. 2019, A\&A, 629, A145

Gauza, B., Bejar, V. J. S., Pérez-Garrido, A., et al. 2015, ApJ, 804, 96

Ge, H., Zhang, X., Fletcher, L. N., et al. 2019, AJ, 157, 89

Gillon, M., Triaud, A. H. M. J., Jehin, E., et al. 2013, A\&A, 555, L5

Ireland, M. J., Kraus, A., Martinache, F., Law, N., \& Hillenbrand, L. A. 2011, ApJ, 726, 113

Kraus, A. L., Ireland, M. J., Cieza, L. A., et al. 2014, ApJ, 781, 20

Kümmel, M., Walsh, J. R., \& The, N. P. P. o. 2009, PASP, 121, 59

Lew, B. W. P., Apai, D., Zhou, Y., et al. 2016, ApJL, 829, L1

Lew, B. W. P., Apai, D., Zhou, Y., et al. 2020, AJ, 159, 125

Manjavacas, E., Apai, D., Lew, B. W. P., et al. 2019, ApJL, 875, L15

Manjavacas, E., Apai, D., Zhou, Y., et al. 2017, AJ, 155, 11

Marley, M. S., Saumon, D., \& Goldblatt, C. 2010, ApJL, 723, L117

Metchev, S. A., Heinze, A., Apai, D., et al. 2015, ApJ, 799, 154

Miles, B. E., Skemer, A. J., Barman, T. S., Allers, K. N., \& Stone, J. M. 2018, ApJ, 869, 18

Miles-Paez, P. A., Metchev, S., Apai, D., et al. 2019, ApJ, 883, 181

Morley, C. V., Marley, M. S., Fortney, J. J., \& Lupu, R. 2014, ApJL, 789, L14

Naud, M.-E., Artigau, E., Rowe, J. F., et al. 2017, AJ, 154, 138

Pearce, L. A., Kraus, A. L., Dupuy, T. J., et al. 2019, AJ, 157, 71

Radigan, J. 2014, ApJ, 797, 120

Radigan, J., Jayawardhana, R., Lafrenière, D., et al. 2012, ApJ, 750, 105

Radigan, J., LaFreniere, D., Jayawardhana, R., \& Artigau, E. 2014, ApJ, 793, 75

Rich, E. A., Currie, T., Wisniewski, J. P., et al. 2016, ApJ, 830, 114

Showman, A. P., \& Kaspi, Y. 2013, ApJ, 776, 85

Stone, J. M., Skemer, A. J., Kratter, K. M., et al. 2016, ApJL, 818, L12

Vos, J. M., Allers, K. N., \& Biller, B. A. 2017, ApJ, 842, 78

Vos, J. M., Biller, B. A., Bonavita, M., et al. 2019, MNRAS, 483, 480

Yang, H., Apai, D., Marley, M. S., et al. 2015, ApJL, 798, L13

Yang, H., Apai, D., Marley, M. S., et al. 2016, ApJ, 826, 8

Zhou, Y., Apai, D., Lew, B. W. P., et al. 2019, AJ, 157, 128

Zhou, Y., Apai, D., Lew, B. W. P., \& Schneider, G. 2017, AJ, 153, 243

Zhou, Y., Apai, D., Metchev, S., et al. 2018, AJ, 155, 132

Zhou, Y., Apai, D., Schneider, G. H., Marley, M. S., \& Showman, A. P. 2016, ApJ, 818, 176 